\title{
Hematopoietic stem cell transplantation for isolated extramedullary relapse of acute lymphoblastic leukemia in children
}

\author{
Maria Gabelli ${ }^{1}{ }^{1} \cdot$ Marco Zecca $^{2} \cdot$ Chiara Messina $^{1} \cdot$ Elisa Carraro ${ }^{1} \cdot$ Barbara Buldini $^{1} \cdot$ Attilio Maria Rovelli $^{3}$. \\ Franca Fagioli ${ }^{4}$ - Alice Bertaina ${ }^{5}$ - Edoardo Lanino ${ }^{6}$. Claudio Favre ${ }^{7}$ - Marco Rabusin ${ }^{8}$. Arcangelo Prete ${ }^{9}$. \\ Mimmo Ripaldi $^{10} \cdot$ Walter Barberi $^{11}$. Fulvio Porta ${ }^{12} \cdot$ Maurizio Caniglia $^{13}$. Stella Santarone ${ }^{14} \cdot$ Paolo $^{\prime}$ Angelo $^{15}$. \\ Giuseppe Basso ${ }^{1} \cdot$ Franco Locatelli ${ }^{5,16}$
}

Received: 14 December 2017 / Revised: 9 May 2018 / Accepted: 31 May 2018 / Published online: 13 June 2018

(c) Macmillan Publishers Limited, part of Springer Nature 2018

\begin{abstract}
Relapse of acute lymphoblastic leukemia (ALL) may occur in extramedullary sites, mainly central nervous system (CNS) and testis. Optimal post-remissional treatment for isolated extramedullary relapse (IEMR) is still controversial. We collected data of children treated with hematopoietic stem cell transplantation (HSCT) for ALL IEMR from 1990 to 2015 in Italy. Among 281 patients, 167 had a relapse confined to CNS, 73 to testis, 14 to mediastinum, and 27 to other organs. Ninetyseven patients underwent autologous HSCT, 79 received allogeneic HSCT from a matched family donor, 75 from a matched unrelated donor, and 30 from an HLA-haploidentical donor. The 10-year overall survival was $56 \%$ and was not influenced by gender, ALL blast immune-phenotype, age, site of relapse, duration of first remission, and type of HSCT. In multivariable analysis, the only prognostic factors were disease status at HSCT and year of transplantation. Patients transplanted in third or subsequent complete remission (CR) had a risk of death 2.3 times greater than those in CR2. Children treated after 2000 had half the risk of death than those treated before that year. Our results suggest that both autologous and allogeneic HSCT may be considered for the treatment of pediatric ALL IEMR after the achievement of CR2.
\end{abstract}

Maria Gabelli

maria.gabelli@aopd.veneto.it

1 Oncoematologia Pediatrica, Università di Padova, Padova, Italy

2 Oncoematologia Pediatrica, Fondazione I.R.C.C.S. Policlinico San Matteo, Pavia, Italy

3 Ematologia Pediatrica, Ospedale San Gerardo, Monza, Italy

4 Oncoematologia Pediatrica, Ospedale Regina Margherita, Azienda Ospedaliero Universitaria, Torino, Italy

5 Oncoematologia Pediatrica, I.R.C.C.S. Ospedale Pediatrico Bambino Gesù, Roma, Italy

6 Centro Trapianti Midollo Osseo, I.R.C.C.S. Giannina Gaslini, Genova, Italy

7 Oncoematologia Pediatrica, Azienda Ospedaliero Universitaria Meyer, Firenze, Italy

8 Oncoematologia Pediatrica, I.R.C.C.S. Materno Infantile Burlo Garofalo, Trieste, Italy
9 Oncologia ed Ematologia Pediatrica "Lalla Seragnoli", Policlinico Sant'Orsola Malpighi, Bologna, Italy

10 Trapianto di Midollo Osseo, Azienda Ospedaliera Santobono Pausilipon, Napoli, Italy

11 Divisione di Ematologia, Università La Sapienza, Roma, Italy

12 Oncoematologia Pediatrica e Trapianto di Midollo Osseo Pediatrico, Spedali Civili di Brescia, Brescia, Italy

13 Oncoematologia Pediatrica, Azienda Ospedaliera di Perugia, Perugia, Italy

14 Dipartimento di Ematologia, Ospedale di Pescara, Pescara, Italy

15 Oncoematologia Pediatrica, A.R.N.A.S. Civico, Di Cristina e Benfratelli, Palermo, Italy

16 Dipartimento di Scienze Pediatriche, Università di Pavia, Pavia, Italy 


\section{Introduction}

Although current treatment protocols cure up to $85 \%$ of children affected by acute lymphoblastic leukemia (ALL), relapse is still the leading cause of treatment failure, affecting $15-20 \%$ of patients. Leukemia relapse may occur in extramedullary sites, mainly central nervous system (CNS) and testis, either alone or in combination with bone marrow (BM) relapse [1].

Site of relapse and duration of first remission are the most important prognostic factors in relapsed ALL, early and isolated BM relapse predicting the worst outcome [2, 3]. While the benefit of allogeneic hematopoietic stem cell transplantation (allo-HSCT) has been demonstrated for high-risk relapsed patients, optimal post-remissional treatment for low-risk patients is still controversial [4-8]. Our previous studies $[9,10]$ demonstrated that autologous HSCT (auto-HSCT) may be a good curative option for children experiencing isolated extramedullary relapse (IEMR). The observation that the immune-surveillance exerted by the allograft against leukemia (graft-versus-leukemia, GVL, effect) is more effective in preventing BM relapse than IEMR [11], led us to hypothesize that the agents used in the conditioning regimen (including total body irradiation, TBI) may be sufficient for disease control in patients with IEMR. This approach may reduce the toxicity associated with allo-HSCT and largely related to graft-versus-host disease (GVHD) occurrence.

Therefore, to further address the role of auto- and alloHSCT in patients experiencing IEMR, we analyzed data of a large cohort of children with first or subsequent ALL IEMR treated with HSCT over a 25 -year period in Italy. To the best of our knowledge, this is the largest study that uniformly analyzes the outcome of this subgroup of patients.

\section{Patients and methods}

This is a retrospective multicenter study involving 20 Italian centers affiliated to the Italian Pediatric Onco-Hematology Association (AIEOP) network.

Data were extracted from the AIEOP-Stem Cell Transplantation (AIEOP-SCT) Registry. We included children (age 1-18 years) with ALL IEMR who underwent HSCT between 1st of January 1990 and 31st of December 2015. Follow-up was updated on January 30th, 2018.

Written informed consent was obtained from parents or legal guardians.

Patients were treated according to the national protocols available at that time, based on Berlin-Frankfurt-Münster (BFM) Study Group backbone, which received approval by the ethical committee of each center.
IEMR was defined as the presence of lymphoblasts in extramedullary sites with less than $5 \%$ blasts in BM. CNS relapse was defined as the presence of $>5$ cells $/ \mu \mathrm{L}$ in the cerebrospinal fluid (CSF) and detection of lymphoblasts by CSF cytomorphology, or alternatively, by clinical or radiological signs. Relapse involving testis or other organs was confirmed by biopsy.

"Very early" relapse was defined when disease recurred less than 18 months from primary diagnosis, "early" when disease recurred later than 18 months from diagnosis and less than 6 months from treatment discontinuation, and "late" when disease recurred more than 6 months from treatment discontinuation [3].

HSCT was performed in patients with second or subsequent complete remission (CR), or, in a limited number of cases, with active disease. If an HLA-matched family donor (MFD) was available, allo-HSCT was performed. If not, auto-HSCT, HSCT from a matched unrelated donor (MUD), or haploidentical (haplo-HSCT) were considered. This decision was taken by the single center.

\section{Statistical analysis}

Overall survival (OS) was defined as the time from transplantation to either last follow-up or death due to any cause; whereas disease-free survival (DFS) as the time from transplantation to either last follow-up or disease recurrence or death due to any cause, whichever occurred first. Relapse-free survival (RFS) was defined as the time from transplantation to documented relapse of ALL. Cumulative incidence (CI) of treatment-related mortality (TRM) was defined as the time from transplantation to death from causes other than disease recurrence/progression, considering relapse as the competing event.

OS, DFS, and RFS were calculated at 10 years using the Kaplan-Meier method with standard error (SE); difference in survival between groups was estimated through the logrank test.

CI of TRM was evaluated at 100 days, 6 months, 1 year, and 10 years after transplantation. Incidence curves were compared using the Gray's test. In multivariable Cox regression analysis, all factors with a $p$-value $<0.2$ in univariable analysis were included. The risk of death was expressed as the hazard ratio (HR) with $95 \%$ confidence interval. Differences in the distribution of various parameters were compared using Chi-square or Fisher exact test as appropriate. A two-sided $p$-value $<0.05$ was considered statistically significant.

The analysis was performed with SAS software (SASPC, version 9.3, SAS Institute, Cary, NC). 


\section{Results}

\section{Patient characteristics}

Two hundred and ninety-two children with IEMR of ALL underwent HSCT from 1990 to 2015 in Italy. Patients included in the study were 281,11 children were excluded because of insufficient data. Patients' characteristics are detailed in Table 1, while conditioning regimens are listed in Table 2. Mean follow-up from transplantation was 6.9 years (median 4.4 years, range $0.03-25.8$ years).

\section{Outcome}

Eighty-three out of 281 patients (29.5\%) experienced a second relapse or disease progression at a median time of 176 days (range 15-2345) from HSCT: 49 patients had an isolated BM recurrence, 16 an IEMR, and 7 a combined relapse; the site of recurrence was unknown in 11 patients. One hundred and eighteen patients (42.0\%) died at a median time of 219 days (range 12-6623) from HSCT: 63 from relapse, 46 from treatment-related complications (14/ 46 were in relapse), 4 from a second tumor, 5 from an unknown event. Grade II-IV acute GVHD (aGVHD) occurred in 79 of 184 patients (42.9\%) who received an allograft, while chronic GVHD (cGVHD) was diagnosed in 32 out of 151 patients $(21.2 \%)$ alive at day +100 after alloHSCT.

\section{Overall survival}

The OS for the entire cohort was $56 \pm 3 \%$ at 10 years; it was not influenced by gender, ALL blast immunephenotype (B-cell precursor [Bcp]-ALL vs T-ALL), age ( $\leq 10$ years vs $>10$ years), site of relapse, source of stem cells, use of TBI during the conditioning regimen and length of first CR (10-year OS for very early, early, and late IEMR was $52 \pm 6 \%, 53 \pm 5 \%$, and $61 \pm 6 \%$, respectively, $p$ $=0.39$ ). No statistically significant difference was observed if a different type of HSCT were compared: OS for auto-HSCT, MFD, MUD, and haplo-HSCT was $57 \pm$ $5 \%, 56 \pm 6 \%, 62 \pm 6 \%$, and $46 \pm 10 \%$, respectively, $p=$ 0.09 (Fig. 1).

In univariable analysis, the prognostic factors associated with OS were: remission status at transplantation and the year in which patients were treated. Patients transplanted in CR2 had a better OS at 10 years $(64 \pm 4 \%)$, in comparison to both those transplanted in subsequent $\mathrm{CR}(\mathrm{CR}>2)$ who showed an OS of $44 \pm 7 \%$ and patients transplanted with active disease who had an OS of $11 \pm 7 \%(p<0.0001)$ (Fig. 2). For patients given HSCT before 2000, the 10-year OS was $45 \pm 5 \%$, while that of children transplanted after 2000 was $63 \pm 4 \%(p=0.0009)$.

\section{Disease-free survival}

The 10 -year-DFS for the whole cohort was $54 \pm 3 \%$. DFS did not differ in relation to gender, ALL blast immunephenotype, age, duration of first CR, type of HSCT, or stem cell source. As for site of relapse, DFS was slightly better for patients with isolated testicular relapse $(65 \pm 6 \%)$ compared to CNS relapse $(49 \pm 4 \%)$, CNS relapse together with other sites $(55 \pm 15 \%)$, mediastinal relapse $(40 \pm 14 \%)$, and other sites involvement $(65 \pm 13 \%)$, but this difference was not statistically significant $(p=0.22)$.

Factors influencing DFS were: presence of TBI in the conditioning regimen, remission status at HSCT, and year of transplantation. TBI-containing regimens were associated with a better DFS compared to non-TBI-containing regimens $(58 \pm 4 \%$ vs $37 \pm 8 \%, p=0.008)$. Remission status at HSCT strongly correlated with DFS: patients transplanted in CR2 had a better 10-year-DFS $(63 \pm 4 \%)$ in comparison to those transplanted in $\mathrm{CR}>2(39 \pm 7 \%)$ or not in remission $(11 \pm 7 \%)(p<0.0001)$. DFS for patients transplanted either before or after 2000 was $45 \pm 5 \%$ and $61 \pm 4 \%$, respectively $(p=0.0008)$.

\section{Transplant-related mortality}

TRM for the entire cohort was $10 \pm 2 \%$ at 100 days, $11 \pm 2 \%$ both at 6 months and 1 year, and $16 \pm 2 \%$ at 10 years. TRM for auto-HSCT was $4 \pm 2 \%, 6 \pm 2 \%, 6 \pm 2 \%$, and $11 \pm 3 \%$, while TRM for allo-HSCT (MUD, MFD, and haplo-HSCT) was $13 \pm 2 \%, 14 \pm 3 \%, 14 \pm 3 \%$, and $18 \pm 3 \%$ at 100 days, 6 months, 1 year, and 10 years, respectively. Comparison resulted not statistically significant $(p=0.08)$.

No statistical significant difference was observed if TRM of patients transplanted before 2000 was compared to that of patients transplanted after $2000(p=0.33)$. In detail, TRM of patients transplanted before 2000 was $15 \pm 3 \%, 16$ $\pm 4 \%, 17 \pm 3 \%$, and $17 \pm 4 \%$ at 100 days, 6 months, 1 year, and 10 years, respectively. TRM of patients transplanted after 2000 was $6 \pm 2 \%, 8 \pm 2 \%, 8 \pm 2 \%$, and $15 \pm 3 \%$ at 100 days, 6 months, 1 year, and 10 years, respectively.

\section{Subgroup analysis and multivariable analysis}

As length of first $\mathrm{CR}$ is one of the most important prognostic factors in relapsed ALL, we performed separate analyses for patients with very early, early, and late IEMR. Regarding patients experiencing very early relapse $(n=87)$, DFS and OS at 10 years showed a trend in favor of allogeneic HSCT (MFD, MUD, and haplo combined) vs autologous HSCT ( $58 \pm 6 \%$ vs $44 \pm 12 \%$ and $59 \pm 6 \%$ vs 44 $\pm 12 \%, p=0.28$ and 0.29 , respectively) (Fig. 3a). In early relapsed patients $(n=97)$, DFS and OS were comparable irrespectively whether patients were treated with either allo- 
Table 1 Characteristics of 281 children who underwent HSCT for isolated extramedullary relapse of ALL from 1990 to 2015 in Italy

\begin{tabular}{|c|c|c|c|c|c|c|}
\hline Number of pts $(\%)$ & $\begin{array}{l}\text { AUTO-HSCT } \\
(n=97)\end{array}$ & $\begin{array}{l}\text { MFD-HSCT } \\
(n=79)\end{array}$ & $\begin{array}{l}\text { MUD-HSCT } \\
(n=75)\end{array}$ & $\begin{array}{l}\text { Haplo-HSCT } \\
(n=30)\end{array}$ & $\begin{array}{l}\text { Total } \\
(n=281)\end{array}$ & $p$ Value \\
\hline Gender & & & & & & 0.83 \\
\hline Male & $67(69.1 \%)$ & $58(73.4 \%)$ & $55(73.3 \%)$ & $23(76.7 \%)$ & $203(72.3 \%)$ & \\
\hline Female & $30(30.9 \%)$ & $21(26.6 \%)$ & $20(26.7 \%)$ & $7(23.3 \%)$ & $78(27.7 \%)$ & \\
\hline $\begin{array}{l}\text { Median age at relapse, years } \\
\text { (range) }\end{array}$ & $4.9(0.3-15.2)$ & $5.6(1.0-17.8)$ & $5.3(0.4-18.0)$ & $5.8(1.5-11.5)$ & & 0.55 \\
\hline Blast immune-phenotype ${ }^{b}$ & & & & & & $0.003^{\mathrm{a}}$ \\
\hline Bcp & $82(84.5 \%)$ & $59(74.7 \%)$ & $55(73.3 \%)$ & $15(50.0 \%)$ & $211(75.1 \%)$ & \\
\hline $\mathrm{T}$ & $7(7.2 \%)$ & $10(12.6 \%)$ & $15(20.0 \%)$ & $9(30.0 \%)$ & $41(14.6 \%)$ & \\
\hline Other & $2(2.1 \%)$ & $1(1.3 \%)$ & 0 & 0 & $3(1.1 \%)$ & \\
\hline Not known & $6(6.2 \%)$ & $9(11.4 \%)$ & $5(6.7 \%)$ & $6(20.0 \%)$ & $26(9.2 \%)$ & \\
\hline Site of relapse $\mathrm{b}^{\mathrm{b}}$ & & & & & & 0.23 \\
\hline CNS & $57(58.8 \%)$ & $51(64.5 \%)$ & $44(58.7 \%)$ & $15(50.0 \%)$ & $167(59.4 \%)$ & \\
\hline Testis & $34(35.0 \%)$ & $17(21.5 \%)$ & $14(18.7 \%)$ & $8(26.7 \%)$ & $73(26.0 \%)$ & \\
\hline Mediastinum & $1(1.0 \%)$ & $2(2.6 \%)$ & $8(10.7 \%)$ & $3(10.0 \%)$ & $14(5.0 \%)$ & \\
\hline $\mathrm{CNS}+$ other & $2(2.1 \%)$ & $3(3.8 \%)$ & $5(6.6 \%)$ & $1(3.3 \%)$ & $11(3.9 \%)$ & \\
\hline CNS + cerebral parenchima & 1 & 0 & 2 & 1 & 4 & \\
\hline CNS + testis & 0 & 2 & 1 & 0 & 3 & \\
\hline CNS + mediastinum & 0 & 0 & 2 & 0 & 2 & \\
\hline$C N S+$ eye & 1 & 1 & 0 & 0 & 2 & \\
\hline Other & $3(3.1 \%)$ & $6(7.6 \%)$ & $4(5.3 \%)$ & $3(10.0 \%)$ & $16(5.7 \%)$ & \\
\hline Eye & 0 & 3 & 0 & 1 & 4 & \\
\hline Lymph-nodes & 1 & 1 & 0 & 1 & 3 & \\
\hline $\begin{array}{l}\text { Other sites (liver, ovary, kidney, } \\
\text { skin...) }\end{array}$ & 2 & 2 & 4 & 1 & 9 & \\
\hline Time to relapse ${ }^{\mathrm{b}}$ & & & & & & $0.004^{\mathrm{a}}$ \\
\hline Very early & $16(16.5 \%)$ & $27(34.2 \%)$ & $33(44.0 \%)$ & $11(36.7 \%)$ & $87(31.0 \%)$ & \\
\hline Early & $33(34.0 \%)$ & $28(35.4 \%)$ & $26(34.7 \%)$ & $10(33.3 \%)$ & $97(34.5 \%)$ & \\
\hline Late & $42(43.3 \%)$ & $21(26.6 \%)$ & $16(21.3 \%)$ & $8(26.7 \%)$ & $87(31.0 \%)$ & \\
\hline not known & $6(6.2 \%)$ & $3(3.8 \%)$ & 0 & $1(3.3 \%)$ & $10(3.5 \%)$ & \\
\hline Remission status at HSCT & & & & & & $0.003^{\mathrm{a}}$ \\
\hline CR2 & $78(80.4 \%)$ & $58(73.4 \%)$ & $56(74.7 \%)$ & $12(40.0 \%)$ & $204(72.6 \%)$ & \\
\hline $\mathrm{CR}>2$ & $13(13.4 \%)$ & $16(20.3 \%)$ & $15(20.0 \%)$ & $15(50.0 \%)$ & $59(21.0 \%)$ & \\
\hline Active disease & $6(6.2 \%)$ & $5(6.3 \%)$ & $4(5.3 \%)$ & $3(10.0 \%)$ & $18(6.4 \%)$ & \\
\hline TBI-based conditioning ${ }^{\mathrm{b}}$ & & & & & & 0.056 \\
\hline Yes & $82(84.5 \%)$ & $71(89.9 \%)$ & $55(73.3 \%)$ & $27(90.0 \%)$ & $235(83.6 \%)$ & \\
\hline No & $14(14.5 \%)$ & $7(8.9 \%)$ & $18(24.0 \%)$ & $3(10.0 \%)$ & $42(15.0 \%)$ & \\
\hline Not known & $1(1.0 \%)$ & $1(1.2 \%)$ & $2(2.7 \%)$ & 0 & $4(1.4 \%)$ & \\
\hline Stem cell source ${ }^{b}$ & & & & & & $<0.0001^{\mathrm{a}}$ \\
\hline $\mathrm{BM}$ & $60(61.9 \%)$ & $71(89.9 \%)$ & $52(69.4 \%)$ & $7(23.3 \%)$ & $190(67.6 \%)$ & \\
\hline $\mathrm{CB}$ & 0 & $2(2.5 \%)$ & $17(22.6 \%)$ & $1(3.3 \%)$ & $20(7.1 \%)$ & \\
\hline PBSC & $36(37.1 \%)$ & $3(3.8 \%)$ & $6(8.0 \%)$ & $22(73.4 \%)$ & $67(23.9 \%)$ & \\
\hline $\mathrm{BM}+$ other & $1(1.0 \%)$ & $3(3.8 \%)$ & 0 & 0 & $1(0.4 \%)$ & \\
\hline Year of HSCT & & & & & & $<0.0001^{\mathrm{a}}$ \\
\hline 1990-2000 & $57(58.8 \%)$ & $37(46.8 \%)$ & $7(9.3 \%)$ & $6(20 \%)$ & $107(38.1 \%)$ & \\
\hline 2000-2015 & $40(41.2 \%)$ & $42(53.2 \%)$ & $68(90.7 \%)$ & $24(80 \%)$ & $174(61.9 \%)$ & \\
\hline
\end{tabular}

Abbreviations: Auto autologous, $B c p$ B cell precursor, $B M$ bone marrow, $C B$ cord blood, $C N S$ central nervous system, $C R$ complete remission, haplo haploidentical, $H S C T$ hematopoietic stem cell transplantation, $M F D$ matched family donor, $M U D$ matched unrelated donor, $n$ number, $P B$ peripheral blood, $P B S C$ peripheral blood stem cells, pts patients, $T B I$ total body irradiation

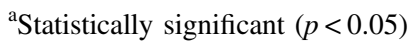

${ }^{b}$ Analysis of significance was performed among most representative groups: Immune-phenotype (T vs Bcp), site of relapse (CNS vs testis), time to relapse (very early vs early and late), TBI-based conditioning (Yes vs No), stem cell source (BM vs CB vs PBSC) 
Table 2 Conditioning regimens

\begin{tabular}{ll}
\hline Conditioning regimen & Number of patients $(\%)$ \\
\hline Cyclo + Thiotepa + TBI & $52(18.5 \%)$ \\
Ara-c + TBI & $44(15.7 \%)$ \\
Thiotepa + Cyclo + ATG + TBI & $24(8.5 \%)$ \\
Etoposide + TBI & $18(6.4 \%)$ \\
Vincristine + Cyclo + TBI & $18(6.4 \%)$ \\
Etoposide + Cyclo + TBI & $14(5.0 \%)$ \\
Thiotepa + Fludara + TBI & $13(4.6 \%)$ \\
Cyclo + TBI & $10(3.6 \%)$ \\
Thiotepa + L-Pam + TBI & $10(3.6 \%)$ \\
Others + TBI & $36(12.8 \%)$ \\
NON-TBI & $42(14.9 \%)$ \\
Bus + Thiotepa + Cyclo & 10 \\
Bus + Cyclo & 5 \\
Bus + Thiotepa + Fludara & 4 \\
Other & 23 \\
\hline
\end{tabular}

Abbreviations: Ara-C Cytarabine, $A T G$ anti-thymocyte globulin, Bus Busulphan, Cyclo Cyclophosphamide, Fludara Fludarabine, L-Pam Melphalan, TBI total body irradiation

or auto-HSCT $(50 \pm 7 \%$ vs $55 \pm 9 \%, p=0.88$ and $52 \pm 7 \%$ vs $54 \pm 9 \%, p=0.87$ ) (Fig. 3b). In late relapses $(n=87)$, DFS and OS were slightly better with auto-HSCT than with allo-HSCT: $65 \pm 8 \%$ vs $48 \pm 9 \%$ and $68 \pm 7 \%$ vs $52 \pm 9 \%$, respectively (Fig. 3c). However, the difference was not statistically significant ( $p=0.13$ and 0.12 , respectively).

Remission status at transplantation is well known to influence the outcome; thus, we conducted a separate analysis for patients in CR2 at time of HSCT $(n=204)$. RFS and OS for this cohort were $74 \pm 3 \%$ and $64 \pm 3 \%$, respectively; outcome of patients given either autologous or

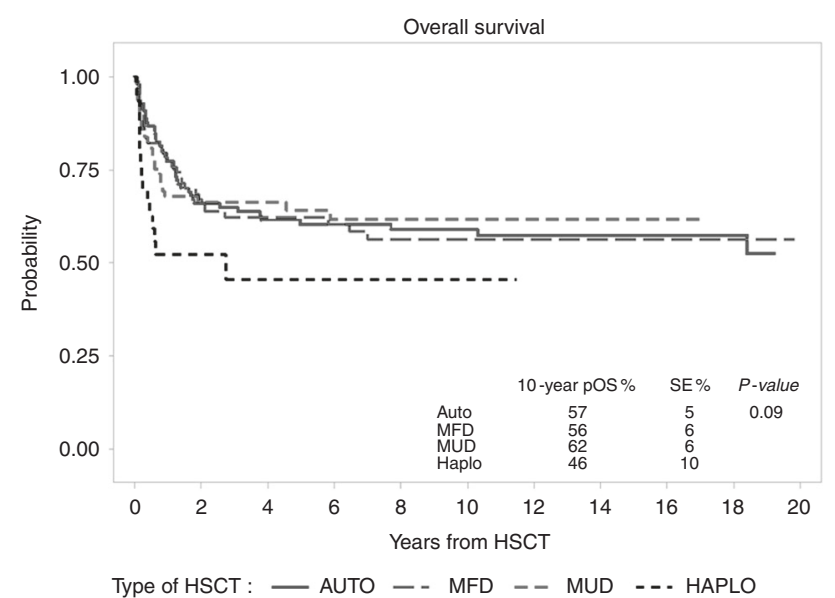

Fig. 1 Overall survival of patients transplanted for extramedullary relapse of ALL according to the type of HSCT employed. Abbreviations: ALL acute lymphoblastic leukemia, Auto autologous, Haplo HLA-haploidentical donor, HSCT hematopoietic stem cell transplantation, MFD matched family donor, MUD matched unrelated donor

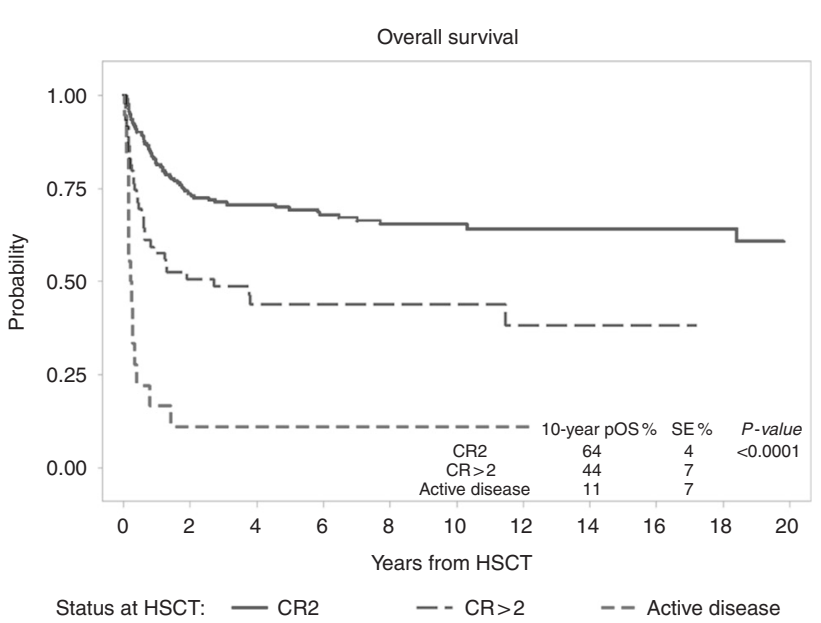

Fig. 2 Overall survival of patients transplanted for extramedullary relapse of ALL: stratification per remission status at HSCT. Abbreviations: ALL acute lymphoblastic leukemia, CR complete remission, HSCT hematopoietic stem cell transplantation

allogeneic HSCT was similar. Ten-year RFS of patients transplanted in CR2 after the year 2000 was better as compared to that of patients transplanted before $2000(79 \pm$ $4 \%$ vs $64 \pm 6 \%$, respectively, $p=0.009$ ).

Since TBI is regarded as the standard regimen conditioning in ALL, we analyzed separately the group of patients who received TBI: 10 -year-DFS did not differ regarding the type of transplant (auto vs allo: $61 \pm 5 \%$ vs $58 \pm 4 \%, p=0.67$ ).

A separate analysis on patients transplanted in more recent years (from 2000 to 2015) was also performed. Results confirmed what we observed analyzing the whole cohort of patients: 10-year OS and DFS were not influenced by site of relapse, presence of TBI, time of relapse, and type of HSCT. Ten-year OS for auto, MFD, MUD, and haploHSCT were $71 \pm 7 \%, 63 \pm 9 \%, 66 \pm 6 \%$, and $46 \pm 13 \%$ ( $p$ $=0.18$ ). Remission status at transplantation was, again, the only variable influencing outcome: OS was $71 \pm 4 \%$ for patients in CR2, $46 \pm 9 \%$ for those in CR $>2(p<0.0001)$; DFS was $69 \pm 4 \%$ and $45 \pm 9 \%(p<0.0001)$, respectively.

For patients treated with allo-HSCT, occurrence of aGVHD was associated with a better DFS $(74 \pm 6 \%$ vs $48 \pm$ $7 \%, p=0.0008)$ and a better OS (63 $\pm 5 \%$ vs $46 \pm 7 \%, p=$ 0.028). Considering only patients given an allograft in CR, occurrence of aGVHD conferred a better RFS: $76 \pm 5 \%$ vs $58 \pm 6 \%, p=0.009$. BM RFS was $87 \pm 4 \%$ for patients who did experience aGVHD vs $74 \pm 6 \%$ for those who did not $(p=0.02)$; conversely, extra-medullary RFS was not affected by aGVHD occurrence $(90 \pm 4 \%$ vs $89 \pm 5 \%, p=$ 0.79). The presence of cGVHD did not influence patients' outcome (data not shown).

Multivariable analysis was conducted after adjustment for remission status: patients with active disease at transplantation were excluded due to the high incidence of 


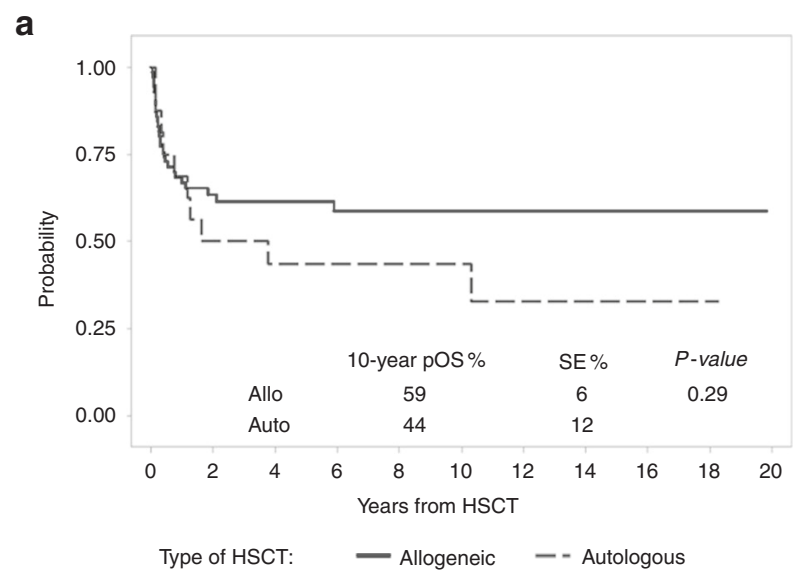

b

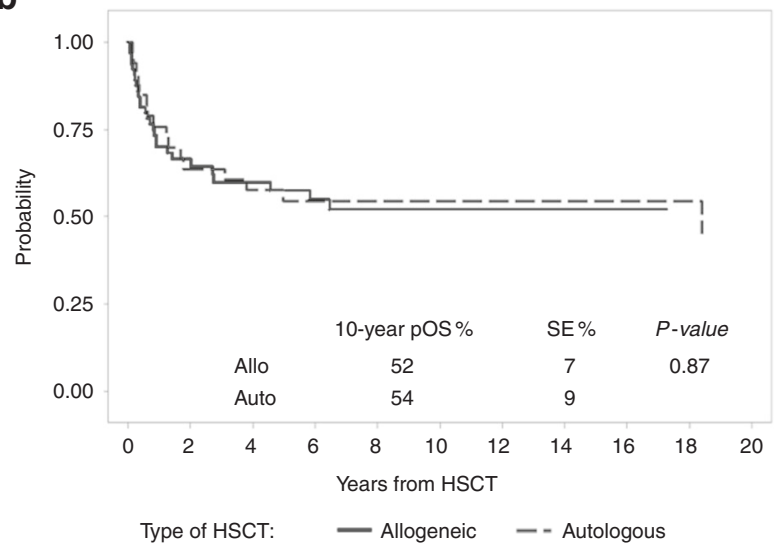

C

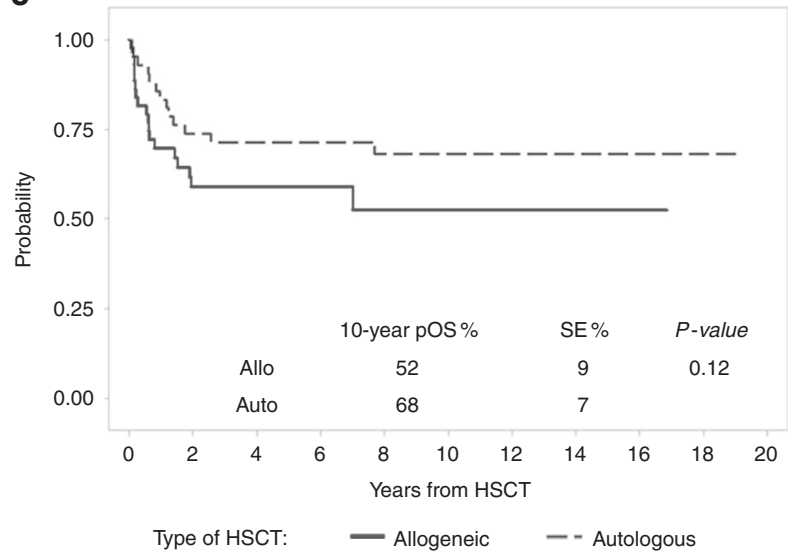

Fig. 3 Overall survival of patients with very early (a), early (b), and late (c) isolated extramedullary ALL relapse: auto-HSCT vs alloHSCT (MFD, MUD, and haplo-HSCT combined). Abbreviations: ALL acute lymphoblastic leukemia, allo-HSCT allogeneic hematopoietic stem cell transplantation, auto-HSCT autologous hematopoietic stem cell transplantation, haplo HLA-haploidentical donor, MFD matched family donor, MUD matched unrelated donor

treatment failure in this group. As shown in Table 3, in multivariable analysis, the only factors influencing OS in patients with IEMR treated with HSCT were number of relapses and year of transplantation.

\section{Discussion}

Although the vast majority of children affected by ALL are cured with current protocols, relapses still occur and pose remarkable challenges to pediatric hematologists. AlloHSCT is currently used to treat patients in CR2 with highrisk features (very early/early and isolated BM relapse, recurrence of T-lineage ALL $[1,12])$, and it is now considered the standard of care also for low-risk patients who present minimal residual disease (MRD) positivity at the end of induction therapy $[13,14]$. Treatment of extramedullary relapse is less well established. The absence of $\mathrm{BM}$ involvement is traditionally considered a favorable prognostic feature [15], and patients with isolated CNS (ICNS) relapse are treated with intensive systemic and intrathecal chemotherapy (CT), followed by either craniospinal or cranial radiotherapy (RT) [5, 7, 16, 17]. EFS with this approach ranges from $45 \%[3,7,18,19]$ to $70 \%$ [16]. Despite the high cure rate obtained in two Children Oncology Group trials [16, 20], with global 5-year EFS approaching $70 \%$, for particular subgroups of IEMR prognosis is still dismal. Patients experiencing very early and early IEMR or ICNS relapses have a survival probability of only $20-30 \%$ in most studies $[3,7,18,19]$. HSCT has been used for treatment of IEMR, but published data are conflicting and limited to small numbers of patients [6, 21-23]. Our previous work [9] showed that EFS of children with early IEMR treated with auto-HSCT was clearly superior to that of patients who received CT/RT (56\% vs $12 \%$ ). Moreover, in another report, we demonstrated that autoHSCT offers a better chance to cure patients in CR2 than in subsequent CR [10]. More recent papers reported comparable outcome in patients with ICNS relapse in CR2 treated with allo-HSCT or CT/RT $[2,5,7]$.

In this study, we present the largest cohort of patients with morphologically defined IEMR of ALL and the largest number of HSCT ever performed for this indication, with a long follow-up (up to 26 years from HSCT). In our cohort, 10-year OS and DFS were around 60\% with either autologous, MFD and MUD-HSCT. Even if a control group of patients treated with CT/RT was not included in this study, our results are comparable with the literature, as reported OS with CT/RT is $45-70 \%$ [3, 5, 7, 16-18, 20]. Moreover, if only patients transplanted in CR2 were considered, as in other published series, the 10 -year OS of $64 \%$ is in line with the most favorable reports $[16,20]$.

Interestingly, in our study, the use of HSCT seems to abrogate the impact of some "classical" prognostic factors, like site of relapse, duration of first remission, and ALL blast immune-phenotype. Similar results were found in the whole cohort, as well as in the group of patients treated in more recent years (from 2000 to 2015). The only factors influencing outcome resulted to be year of HSCT and 
Table 3 Multivariable analysis of factors influencing outcome in children with isolated extramedullary relapse of ALL

\begin{tabular}{|c|c|c|c|c|c|c|c|}
\hline Characteristics & Categories & Pts $n$ & Events & $\begin{array}{l}10-\mathrm{yr} \\
\text { OS \% } \\
(\mathrm{SE} \%)\end{array}$ & $\begin{array}{l}\text { Univariable } \\
p \text {-value }\end{array}$ & $\begin{array}{l}\text { Multivariable } \\
p \text {-value }\end{array}$ & $\begin{array}{l}\text { Hazard ratio } \\
(95 \% \mathrm{CI})\end{array}$ \\
\hline \multirow[t]{2}{*}{ Age } & $\leq 10 \mathrm{yrs}$ & 215 & 82 & $59(4)$ & 0.50 & - & \\
\hline & $>10 \mathrm{yrs}$ & 48 & 16 & $66(7)$ & & & \\
\hline \multirow[t]{2}{*}{ Gender } & Female & 73 & 25 & $60(6)$ & 0.58 & - & \\
\hline & Male & 190 & 73 & $60(4)$ & & & \\
\hline \multirow{2}{*}{$\begin{array}{l}\text { Blast immune- } \\
\text { phenotype }\end{array}$} & Bcp & 203 & 76 & $61(4)$ & 0.42 & - & \\
\hline & $\mathrm{T}$ & 33 & 9 & $68(9)$ & & & \\
\hline \multirow[t]{2}{*}{ Relapse site } & Testis & 72 & 24 & $67(6)$ & 0.23 & 0.56 & \\
\hline & CNS & 154 & 60 & $58(4)$ & & & \\
\hline \multirow{2}{*}{$\begin{array}{l}\text { TBI in } \\
\text { conditioning } \\
\text { regimen }\end{array}$} & No & 36 & 17 & $\begin{array}{l}43 \\
(10)\end{array}$ & 0.21 & 0.86 & \\
\hline & Yes & 224 & 80 & $60(4)$ & & & \\
\hline \multirow[t]{2}{*}{ Year of HSCT } & Before 2000 & 94 & 49 & $51(5)$ & 0.0064 & 0.0035 & $0.5(0.3-0.8)$ \\
\hline & After 2000 & 169 & 49 & $65(4)$ & & & \\
\hline \multirow[t]{2}{*}{ HSCT type } & Autologous & 91 & 36 & $62(5)$ & 0.63 & - & \\
\hline & Allogeneic & 172 & 62 & $60(4)$ & & & \\
\hline \multirow[t]{2}{*}{ Status at HSCT } & $\mathrm{CR} 2$ & 204 & 65 & $65(4)$ & $<0.0001$ & 0.0005 & $2.3(1.4-30.7)$ \\
\hline & $\mathrm{CR}>2$ & 59 & 33 & $44(7)$ & & & \\
\hline
\end{tabular}

Abbreviations: $B c p$ B cell precursor, $C I$ confidence interval, $C N S$ central nervous system, $C R$ complete remission, HSCT hematopoietic stem cell transplantation, $n$ number, $O S$ overall survival, Pts patients, TBI total body irradiation, yrs years remission status at transplantation. Taking into account the prognostic impact of year of transplantation, this may reflect improvement in patient selection: the 10-year RFS for patients transplanted before 2000 was better than that of patients transplanted after 2000; on the contrary, 10-year TRM pre and post-2000 did not differ. There is the possibility that MRD assessment during therapy guided decisions on CT administration, time and type of HSCT in single centers. As far as the prognostic significance of remission status before HSCT is concerned, these data confirm what we reported previously [10], namely that outcome is significantly better for children transplanted in CR2 than in subsequent remission. This observation emphasizes the importance of identifying those patients at higher risk of further relapse, who, thus, may benefit from HSCT soon after the achievement of CR2. Very early and early IEMR or ICNS relapses treated with CT/RT have been previously shown to have a survival probability around $20-30 \%[3,7$, $18,19]$. The use of HSCT in our study improved the OS to $53 \%$ for early relapses and $52 \%$ for very early relapses. This result is even more relevant considering that patients with third or subsequent $\mathrm{CR}$ and even with active disease at time of HSCT were included in this analysis. Based on these data, we suggest considering HSCT for patients with very early/early IEMR once that the CR is achieved.

Furthermore, no difference in outcome was observed regarding the type of HSCT. This finding is in line with our previous study, where we reported that auto-HSCT had the same chance to cure children with ICNS relapse than MFDHSCT [9]. Present data strengthen this observation, including MUD-HSCT and (although with a low number of cases) haplo-HSCT. The favorable results obtained with either auto and allo-HSCT may be due to the large use of TBI in the conditioning regimen. Moreover, based on published reports $[11,24,25]$, we speculate that the GVL effect of allo-HSCT may be less relevant in extramedullary site, as migration/homing of donor $\mathrm{T}$ cells may be impaired at extramedullary sites. In this regard, it was reported that donor cells are absent in extra-medullary sites of patients who relapsed after HSCT [26, 27]. Furthermore, donor lymphocytes infusion and recent chimeric antigen receptor $\mathrm{T}$ cells have been reported to be less effective in extramedullary disease control [28-30]. In line with these observations and with a previous report [11], in our alloHSCT cohort, occurrence of aGVHD decreased the incidence of subsequent BM relapses but no of subsequent IEMR. Therefore, we can hypothesize that, in the group of patients with IEMR of ALL, those with higher risk of subsequent BM relapse (i.e., children with positive BM MRD) may benefit more from allo-HSCT, while patients with pure IEMR relapse (i.e., negative BM MRD) could be offered auto-HSCT.

This study shows that both auto- and allo-HSCT are effective treatments for IEMR of ALL, to be considered as soon as CR2 is achieved. Patients with late IEMR, currently treated with CT/RT, may benefit from auto-HSCT also in 
terms of shorter treatment duration, resulting in better quality of life for patients and their families. Current Italian strategy to treat children with very early and early IEMR recommend allo-HSCT [13]. This study shows that autoHSCT may be a good alternative, significantly reducing the time patients wait before transplantation and the risk of both GVHD and infection-related mortality/morbidity associated with allo-HSCT. The role of auto-HSCT for patients with late IEMR or very early/early IEM with negative BM MRD remains to be assessed in future trials.

The retrospective nature of this study and the absence of data regarding $\mathrm{BM}$ MRD before transplantation represent significant limitations of our study; however, the large number of patients with IEMR and the long follow-up strengthen our results. The role of auto- and allo-HSCT in the treatment of IEMR of pediatric ALL should be further explored in prospective studies including MRD assessment for stratifying patients.

Acknowledgments We are grateful to Dr. Veronica Tintori (Bone Marrow Transplant Unit, Azienda Ospedaliero Universitaria Meyer, Firenze, Italy) and to Dr. Gabriella Casazza (U.O. Oncoematologia Pediatrica, Azienda Ospedaliero Universitaria di Pisa, Italy) for providing patients' data.

\section{Compliance with ethical standards}

Conflict of interest The authors declare that they have no conflict of interest.

\section{References}

1. Bhojwani D, Pui CH. Relapsed childhood acute lymphoblastic leukemia. Lancet Oncol. 2013;14:e205-17.

2. Malempati S, Gaynon PS, Sather H, La MK, Stork LC. Outcome after relapse among children with standard-risk acute lymphoblastic leukemia: Children's Oncology Group study CCG-1952. J Clin Oncol. 2007;25:5800-7.

3. Tallen G, Ratei R, Mann G, Kaspers G, Niggli F, Karachunsky A, et al. Long-term outcome in children with relapsed acute lymphoblastic leukemia after time-point and site-of-relapse stratification and intensified short-course multidrug chemotherapy: results of trial ALL-REZ BFM 90. J Clin Oncol. 2010;28:2339-47.

4. Jacobs JE, Hastings C. Isolated extramedullary relapse in childhood acute lymphocytic leukemia. Curr Hematol Malig Rep. 2010;5:185-91.

5. Eapen M, Zhang MJ, Devidas M, Raetz E, Barredo JC, Ritchey AK, et al. Outcomes after HLA-matched sibling transplantation or chemotherapy in children with acute lymphoblastic leukemia in a second remission after an isolated central nervous system relapse: a collaborative study of the Children's Oncology Group and the Center for International Blood and Marrow Transplant Research. Leukemia. 2008;22:281-6.

6. Harker-Murray PD, Thomas AJ, Wagner JE, Weisdorf D, Luo X, DeFor TE, et al. Allogeneic hematopoietic cell transplantation in children with relapsed acute lymphoblastic leukemia isolated to the central nervous system. Biol Blood Marrow Transplant. 2008;14:685-92.
7. Domenech C, Mercier M, Plouvier E, Puraveau M, Bordigoni P, Michel G, et al. First isolated extramedullary relapse in children with B-cell precursor acute lymphoblastic leukaemia: results of the Cooprall-97 study. Eur J Cancer. 2008;4:2461-9.

8. van den Berg H,de Groot-Kruseman HA,Damen-Korbijn CM,de Bont ESJM,Schouten-van Meeteren AYN,Hoogerbrugge PM, Outcome after first relapse in children with acute lymphoblastic leukemia: a report based on the Dutch Childhood Oncology Group (DCOG) relapse ALL 98 protocol. Pediatr Blood Cancer. 2011;57:210-6.

9. Messina C, Valsecchi MG, Aricò A, Locatelli F, Rossetti F, Rondelli R, et al. Autologous bone marrow transplantation for treatment of isolated central nervous system relapse of childhood acute lymphoblastic leukemia. Bone Marrow Transplant. 1998;21:9-14.

10. Messina C, Cesaro S, Rondelli R, Rossetti F, Locatelli F, Pession A, et al. Autologous bone marrow transplantation for childhood acute lymphoblastic leukaemia in Italy. AIEOP/FONOP-TMO Group. Italian Association of Paediatric Haemato-Oncology. Bone Marrow Transplant. 1998;21:1015-21.

11. Lee JH, Choi SJ, Lee JH, Seol M, Lee YS, Ryu SG, et al. Antileukemic effect of graft-versus-host disease on bone marrow and extramedullary relapses in acute leukemia. Haematologica. 2005;90:1380-8.

12. Borgmann A, von Stackelberg A, Hartmann R, Ebell W, Klingebiel T, Peters C, et al. Unrelated donor stem cell transplantation compared with chemotherapy for children with acute lymphoblastic leukemia in a second remission: a matched-pair analysis. Blood. 2003;101:3835-9.

13. Locatelli F, Schrappe M, Bernardo ME, Rutella S. How I treat relapsed childhood acute lymphoblastic leukemia. Blood. 2012;120:2807-16.

14. Balduzzi A, Galimberti S, Valsecchi MG, Bonanomi S, Conter V, Barth A, et al. Autologous purified peripheral blood stem cell transplantation compare to chemotherapy in childhood acute lymphoblastic leukemia after low-risk relapse. Pediatr Blood Cancer. 2011;57:654-9.

15. Nguyen K, Devidas M, Cheng SC, La M, Raetz EA, Carroll WL, et al. Factors influencing survival after relapse from acute lymphoblastic leukemia. A Children's Oncology Group study. Leukemia. 2008;22:2142-50.

16. Ritchey AK, Pollock BH, Lauer SJ, Andejeski Y, Buchanan GR. Improved survival of children with isolated CNS relapse of acute lymphoblastic leukemia: a Pediatric Oncology Group study. J Clin Oncol. 1999; 17:3745-52.

17. Einsiedel HG, von Stackelberg A, Hartmann R, Fengler R, Schrappe M, Janka-Schaub G, et al. Long-term outcome in children with relapsed ALL by risk-stratified salvage therapy: results of trial acute lymphoblastic leukemia-relapse study of the BerlinFrankfurt-Munster Group 87. J Clin Oncol. 2005;23:7942-50.

18. Roy A, Cargill A, Love S, Moorman AV, Stoneham S, Lim A, et al. Outcome after first relapse in childhood acute lymphoblastic leukaemia-lessons from the United Kingdom R2 trial. Br J Haematol. 2005;130:67-75.

19. Saarinen-Pihkala UM, Heilmann C, Winiarski J, Glomstein A, Abrahamsson J, Arvidson J, et al. Pathways through relapses and deaths of children with acute lymphoblastic leukemia: role of allogeneic stem cell transplantation in Nordic data. J Clin Oncol. 2006;24:5750-62.

20. Barredo JC, Devidas M, Lauer SJ, Billett A, Marymont MA, Pullen J, et al. Isolated CNS relapse of acute lymphoblastic leukemia treated with intensive systemic chemotherapy and delayed CNS radiation: a Pediatric Oncology Group Study. J Clin Oncol. 2006;24:3142-9.

21. Bordigoni P, Esperou H, Souillet G, Pico J, Michel G, Lacour B, et al. Total body irradiation-high-dose cytosine arabinoside and 
melphalan followed by allogeneic bone marrow transplantation from HLA-identical siblings in the treatment of children with acute lymphoblastic leukaemia after relapse while receiving chemotherapy: a Société Française de Greffe de Moelle study. Br J Haematol. 1998;102:656-65.

22. Afify Z, Hunt L, Green A, Guttridge M, Cornish J, Oakhill A. Factors affecting the outcome of stem cell transplantation from unrelated donors for childhood acute lymphoblastic leukemia in third remission. Bone Marrow Transplant. 2005;35:1041-7.

23. Yoshihara $\mathrm{T}$, Morimoto A, Kuroda $\mathrm{H}$, Imamura $\mathrm{T}$, Ishida $\mathrm{H}$, Tsunamoto K, et al. Allogeneic stem cell transplantation in children with acute lymphoblastic leukemia after isolated central nervous system relapse: our experiences and review of the literature. Bone Marrow Transplant. 2006;37:25-31.

24. Shem-Tov N, Saraceni F, Danylesko I, Shouval R, Yerushalmi R, Nagler A, et al. Isolated extramedullary relapse of acute leukemia after allogeneic stem cell transplantation: different kinetics and better prognosis than systemic relapse. Biol Blood Marrow Transplant. 2017;23:1087-94.

25. Lee K-H, Lee J-H, Kim S, Lee J-S, Kim S-H, Kim W-K. High frequency of extramedullary relapse of acute leukemia after allogeneic bone marrow transplantation. Bone Marrow Transplant. 2000;26:147-52.
26. Huck K, Laws H-J, Meisel R, Tröger A, Bernbeck B, Schönberger $\mathrm{S}$, et al. Three cases of renal relapse after allogeneic hematopoietic stem cell transplantation for childhood acute lymphoblastic leukemia. Haematologica. 2006;91:e20-5.

27. Kantarcioglu B, Bekoz HF, Ogret YD, Cakir A, Kivanc D, Oguz $\mathrm{F}$, et al. Isolated extramedullary cutaneous relapse despite concomitant severe graft-vs.-host disease and tissue chimerism analysis in a patient with acute lymphoblastic leukemia after allogeneic hematopoietic stem cell transplantation: a case report. Mol Clin Oncol. 2016;5:745-9.

28. Singhal S, Powles R, Kulkarni S, Treleaven J, Saso R, Mehta J. Long-term follow-up of relapsed acute leukemia treated with immunotherapy after allogeneic transplantation: the inseparability of graft-versus-host disease and graft-versus-leukemia, and the problem of extramedullary relapse. Leuk Lymphoma. 1999;32:505-12.

29. Neumann M, Blau IW, Burmeister T, Tietze-Buerger C, Blau O, Gerbits A, et al. Intrathecal application of donor lymphocytes in leukemic meningeosis after allogeneic stem cell transplantation. Ann Hematol. 2011;90:911-6.

30. Park JH, Rivière I, Gonen M, Wang Y, Senechal B, Curran KJ, et al. Long-term follow-up of CD19 CAR therapy in acute lymphoblastic leukemia. N Engl J Med. 2018;378:449-59. 